Eastern Illinois University

The Keep

Faculty Research \& Creative Activity

Biological Sciences

January 2008

\title{
Simulating the effects of wetland loss and inter- annual variability on the fitness of migratory bird species
}

\author{
Jill L. Deppe \\ Eastern Illinois University, jldeppe@eiu.edu \\ james A. Smith \\ NASA Goddard Space Flight Center
}

Follow this and additional works at: http://thekeep.eiu.edu/bio_fac

Part of the Biology Commons

\section{Recommended Citation}

Deppe, Jill L. and Smith, james A., "Simulating the effects of wetland loss and inter-annual variability on the fitness of migratory bird species" (2008). Faculty Research \& Creative Activity. 156.

http://thekeep.eiu.edu/bio_fac/156 
From - Proc. IEEE Geosciences and Remote Sensing Symposium, Boston, MA, November 2008

\title{
SIMULATING THE EFFECTS OF WETLAND LOSS AND INTER-ANNUAL VARIABILITY ON THE FITNESS OF MIGRATORY BIRD SPECIES
}

\author{
James A. Smith \\ NASA Goddard Space Flight Center \\ Jill L. Deppe \\ University of Maryland Baltimore County, Goddard Earth Sciences and Technology Center
}

\begin{abstract}
Long-distance migratory shorebirds require wetland stopover sites where they can forage and deposit sufficient fat to complete their migration and, in the spring, reproduce. Conservation biologists are concerned that continental-scale reductions in wetland availability and quality due to human disturbance, climate change, and natural drought events are negatively impacting these species by eliminating critical stopovers along migratory flyways. We describe an individual-based migration model driven by remotely sensed land surface data, climate data assimilation models, and biological field data to examine the impact of changing environmental conditions on migration routes, temporal patterns, and fitness. We used an evolutionary programming approach to evaluate birds' adaptive responses to variation in refueling potential in the landscape. Birds' shifted their migratory routes and extended their stopovers as the mean quality of the landscape decreased and spatial variation in refueling potential increased. We discuss possible applications of our model for understanding inter-annual climate variation, climate change, and wetland loss.
\end{abstract}

Index Terms - Bird migration modeling, avian energetics, satellite remote sensing, evolutionary learning

\section{INTRODUCTION}

Inland wetlands and moist habitats, including shallow freshwater and alkaline wetlands, mudflats, and irrigated agricultural fields, provide critical stopover habitat for migratory shorebirds along their extensive flyways through the central United States, Mexico, and Central and South America. Natural fluctuations in the availability of these wetland stopover sites coupled with anthropogenic destruction of wetlands and anticipated losses due to climate change [13] present migratory birds with a formidable challenge. At least $50 \%$ of shorebird species have experienced population declines [1]; however, the mechanisms underlying these declines are unclear.

Important stopover sites along migratory flyways are limited in number and widely dispersed. Shorebirds' reliance on these geographic links makes them vulnerable to losses of key wetland areas. A major concern of conservation biologists is that reductions in the availability of wetland stopover sites due to human activities, climate change, or increased climate variability (e.g., greater severity or frequency of drought events) could negatively impact migratory bird populations and species by reducing the overall quality of the landscape and increasing spacing among suitable stopovers [9].

We are using individually-based bird simulation models driven by remotely sensed land surface data, near real-time or retrospective climate and hydrologic data assimilation models, and biological field data to forecast the impact of drought, anthropogenic wetland loss, and climate change on the fitness, phenological patterns, and routes of migratory birds [4] [10] [11].

Previous migration models have simulated bird movements through static, artificial landscapes of limited geographic extent driven by sparse weather station data. Such models have limited applicability to ecological problems, such as biodiversity loss, because they do not reflect the complexity of the environments that birds experience while migrating.

\section{OBJECTIVES}

In this paper, we describe the use of remote sensing and climate data in our modeling approach to simulate the spring migration of Pectoral Sandpipers (Calidris melanotos) through the Great Plains and Prairie Pothole regions of the United States and central Canada under various environmental conditions. By integrating an evolutionary 


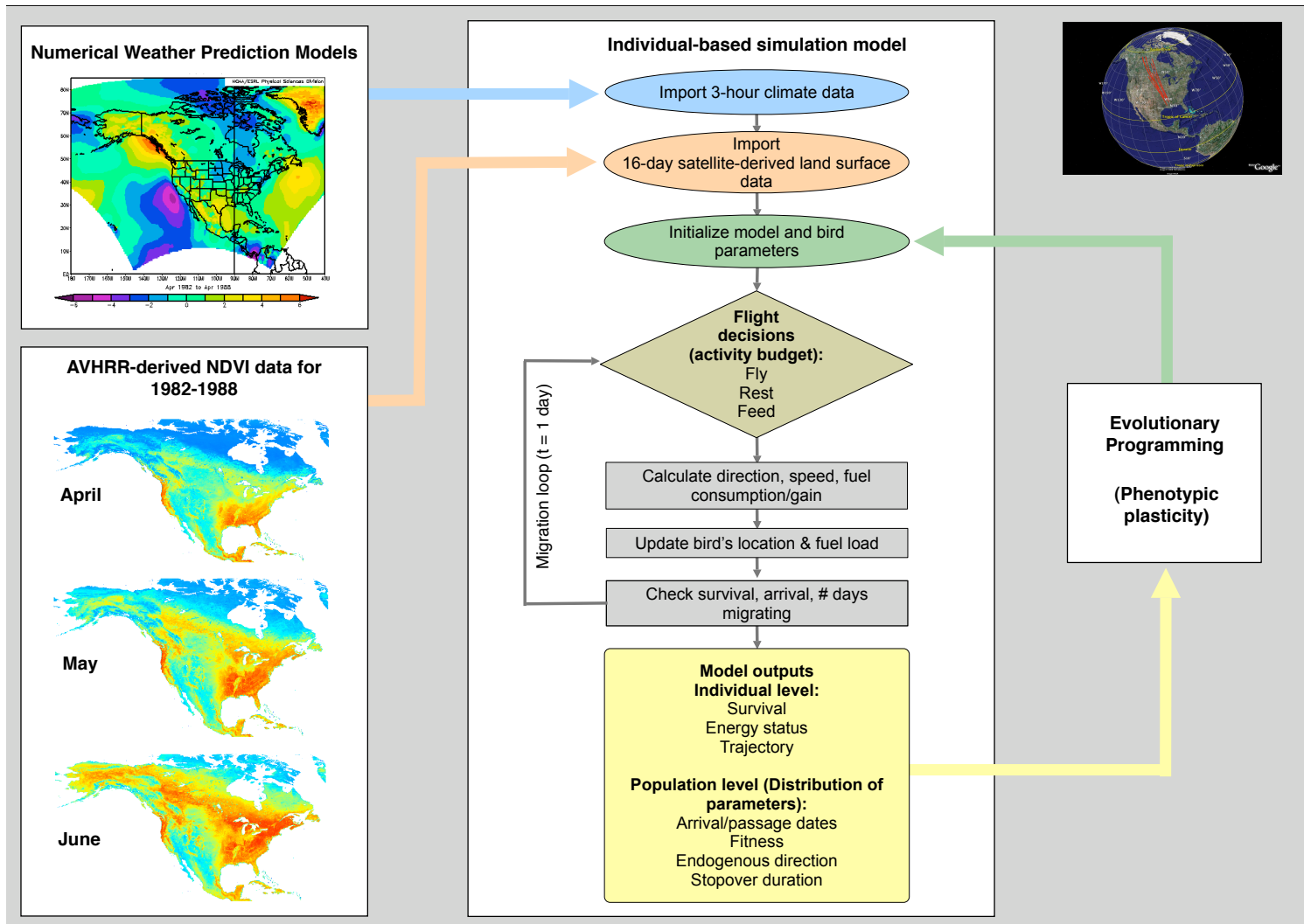

Figure 1. Bird migration model framework illustrating the integration of remote sensing data, near real-time or retrospective climate data assimilation models, biological field data, bird physiological models, and evolutionary programming to predict the migratory routes, timing, stopover behavior, flight direction, and fitness (survival and reproductive success) of migratory birds.

learning algorithm [2] into our migration model we provide an approach for predicting how birds may adapt to these environmental changes over successive generations to maximize their survival and reproductive success.

\section{APPROACH}

Our model is an individual-based, spatially explicit bird migration model [5] that simulates the migration routes, temporal patterns, and energy budgets of individual birds under dynamic weather and land surface conditions. Body fat is the primary source of energy for migratory flights. The amount of fat directly determines a bird's activity budget (i.e., the amount of time allocated to flying, resting, and foraging) and, ultimately, its survival and future reproductive success. The more abundant wetlands and moist habitats are along the migratory flyway, the higher the probability that birds will be able to satisfy their energy requirements in a safe and timely fashion. For females, which we model in our study, the benefits of abundant, high quality en route habitat manifest themselves as higher fat reserves at the time of arrival on the breeding grounds and, thus, higher reproductive output [6]. In addition, evidence suggests that larger fat reserves provide birds with a buffer against en route contingencies, such as inclement weather or inability to locate high quality foraging habitat, thereby increasing their probability of survival.

The speed, direction, and timing of individual birds moving through a user-specified Lagrangian grid depends on (i) the individual bird's energy status, physiology, flight aerodynamics, and stopover behavior and (ii) environmental properties including wind speed and direction, topography, dynamic climate and hydrologic properties of the landscape, and habitat quality (Figure 1). By simulating a population of migrating birds of a species with varying initial body fat, behavioral rules, and inherited characteristics (e.g., flight direction), we can predict migration routes and temporal dynamics of migration intensity, as well as bird status (fat load, location, and survival).

Starting locations and initial fat loads are selected randomly from within empirically defined limits. Similarly, birds are assigned probabilistic endogenous migration directions, e.g. from the wintering to the breeding grounds, based on field observations. We provide the capability to run the model in a calibration mode where unknown parameters can be 
adjusted within known physical and biological constraints until model predictions fit field observations or general patterns.

\subsection{Study Species}

The Pectoral Sandpiper (Calidris melanotos) is a longdistance migratory shorebird that breeds in northern Alaska and Canada and winters in southern South America (Figure 2). During spring migration the species migrates through central North America. The species inhabits shallow wetlands, irrigated agricultural fields, and other moist habitats where it probes the soil in search of invertebrates.

\subsection{Migration Model}

To illustrate our approach we present an example simulation of the spring migration of Pectoral Sandpipers. For each modeling scenario the birds began their northward migration around April 1, just after a trans-gulf migration from Mexico. For successful breeding, the birds must arrive at northern breeding latitudes within a June 1 to June 30 window and with sufficient fat reserves. We randomly selected stopover strategies (i.e., the number of days spent at a stopover site), initial fat load, endogenous flight directions, and starting locations from empirically defined probability distributions.

\subsection{Impact of variable landscapes on bird migration}

We simulated the spring migration of Pectoral Sandpipers through two different landscapes in order to demonstrate how our migration model can be used to understand the potential impacts of changing environmental conditions on the migratory routes, timing, energetic condition, and fitness of long-distance migratory birds. First, we flew birds through a theoretical non-limiting landscape, i.e., constant maximum refueling potential. Then, we flew birds over a landscape of variable refueling potential based on average conditions for 1982-88, a period that encompassed a drought event; the mean refueling potential of this second landscape was less than that of the theoretical non-limiting landscape.

The map of refueling potential during the period of 19821988 was modeled as the product of average monthly AVHRR-derived NDVI (GIMMS data set) [12] and mean number of days of frozen ground per month (IPCC data set) [7]. Refueling potential at a given grid cell was scaled between 0 and 1 . We created maps of spatially variable refueling potential for April, May, and June.

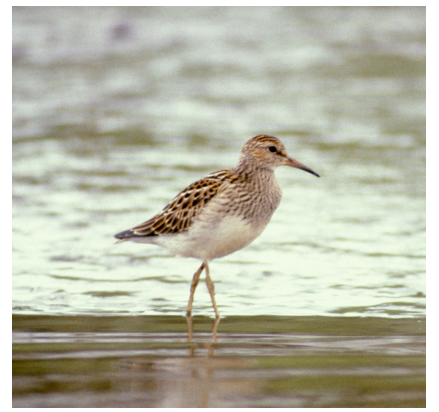

Figure 2. Pectoral Sandpiper (United States Geological Survey photo by Craig Ely).

\subsection{Evolutionary learning algorithm}

We examined sandpipers' adaptive responses to varying environmental conditions by integrating an evolutionary learning algorithm [2] into our migration model and allowing 10 generations of 10000 birds each to fly through the landscape of spatially variable refueling potential. We compared migration routes, phenologies (i.e., timing of passage), mean stopover duration, and mean fitness for the initial and final bird populations to quantify the response of birds to the changed conditions and to illustrate how the algorithm may be used to understand optimal migration strategies for these birds in different environments.

\section{RESULTS}

The population of birds flown through the theoretical nonlimiting landscape had a higher fitness, as expected, and a broader spatial distribution than when flown through a landscape characterized by variable refueling potential.

When populations were allowed to adapt to the landscape of varying refueling potential we observed a shift in migratory routes, in particular birds were less dispersed in the landscape (Figure 3A vs. B). After 10 generations birds appeared to avoid flying over water bodies (northeast Pacific and Hudson Bay) and high elevations, where the likelihood of locating suitable conditions was low. In addition, birds exhibited an increase in mean stopover duration, with an optimum duration of 12.75 days.

\section{CONCLUSIONS}

Our model provides a mechanistic approach for examining the impacts of changing climate and land surface conditions on a wide range of migratory birds, including those that have many potential stopover sites distributed in a more-orless continuous fashion throughout the landscape, e.g., some forest song birds in the eastern United States and Canada. Dynamic state variable models [3] frequently are used to predict optimal migration strategies for migratory birds, but 

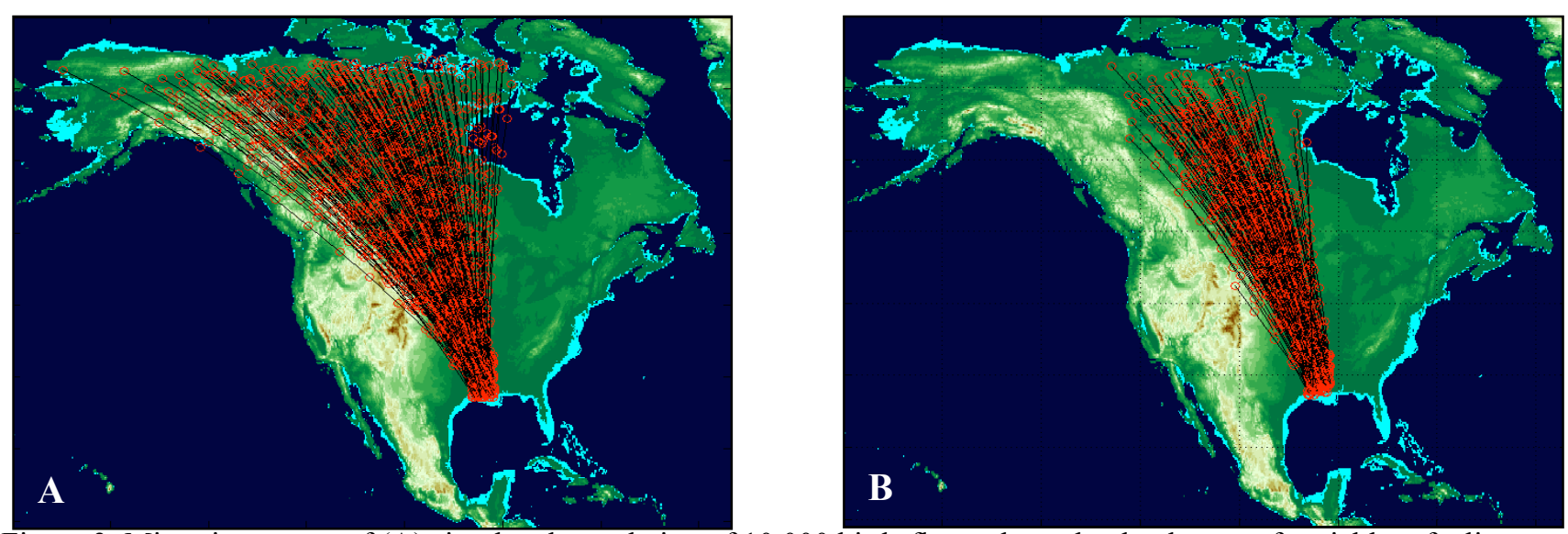

Figure 3. Migration routes of (A) simulated population of 10000 birds flown through a landscape of variable refueling potential with no evolutionary learning and (B) after 10 generations, i.e., with evolutionary learning.

this approach mostly has been applied to species using a relatively small number of discrete stopover sites.

Future research will evaluate the plausibility of our model and its use in applied research by comparing predicted outputs with patterns observed in the field. In addition, we are exploring the use of ecological niche modeling to produce spatio-temporal maps of suitable stopover conditions [8]. By simulating migration through alternative landscapes, as we did in this paper, our model may be used to provide insight into the potential effects of climate change, inter-annual variability (e.g., drought), and anthropogenic wetland loss on birds' migration routes, phenological patterns, survival, and reproductive success.

\section{ACKNOWLEDGEMENTS}

Our research is supported under a NASA IDS research proposal, "Forecasting the effects of wetland loss and interannual variability on the fitness of migratory bird species" and, in part, by a NASA AIST proposal, "A general framework and system prototypes for self-adaptive Earth predictive systems-dynamically coupling sensor web with Earth System models."

\section{REFERENCES}

[1] S. Brown, C. Hickey, B. Harrington, and R. Gill, eds, The U.S. Shorebird Conservation Plan, $2^{\text {nd }} E d$. Manomet, MA, Manomet Center for Conservation Sciences.

[2] K.A. De Jong, Evolutionary computation: a unified approach. (Cambridge MA, MIT Press, 2006).

[3] C.W. Clark and M. Mangel. Dynamic state variable models in ecology. (New York, Oxford University Press., 2000).
[4] J. L. Deppe, K. Wessels, and J. A. Smith. 2007. Alaska at the crossroads of migration: space-based ornithology. Alaska Park Science 6:53-58.

[5] B. Erni, F. Liechti, and B. Bruderer, "How does a first year passerine migrant find its way? Simulating migration mechanisms and behavioural adaptations," OIKOS 103, pp. 333-340, 2003.

[6] A. H. Farmer and John Weins, "Optimal migration schedules depend on the landscape and the physical environment: a dynamic modeling view," J. Avian Biology, 29, pp. 405-415, 1998.

[7] M. New, D. Lister, M. Hulme, and I. Makin. "A high-resolution data set of surface climate over global land areas," Climate Research, 21, pp. 1-25, 2002.

[8] S.J. Phillips, R.P. Anderson, and R.E.Schapire, "Maximum entropy modeling of species geographic distributions," Ecological Modelling 190, pp. 231-259, 2006.

[9] S.K. Skagen, "Migration stopovers and the conservation of Arctic-breeding Calidridine Sandpipers," AUK 123, pp. 313-322, 2006.

[10] J. A. Smith, "Simulation of Avifauna Distributions Using Remote Sensing," Proc. IEEE Int'l Geosci. And Rem. Sens. Symposium. Anchorage, Alaska, 19-24 September 2004.

[11] J. A. Smith and J. L. Deppe, "Simulating Bird Migration using Satellites and Biophysics," Proc. Of the IASTED Symposium on Environmental Modeling and Simulation 509, pp. 6-11, 2007

[12] Tucker, C.J., J.E. Pinzon, and M.E. Brown (2004), Global Inventory Modeling and Mapping Studies, NA94apr15b.n11-VIg, 2.0, Global Land Cover Facility, University of Maryland, College Park, Maryland, 04/15/1994.

[13] T. C. Winter, "The vulnerability of wetlands to climate change: A hydrologic landscape perspective," J. of the American Water Resources Association 36, pp. 305-311, 2000. 